\title{
Fine-grained uncertainty relations for several quantum measurements
}

\author{
Alexey E. Rastegin* \\ Department of Theoretical Physics, Irkutsk State University, Gagarin Bv. 20, Irkutsk 664003, Russia
}

\begin{abstract}
We study fine-grained uncertainty relations for several quantum measurements in a finitedimensional Hilbert space. The proposed approach is based on exact calculation or estimation of the spectral norms of corresponding positive matrices. Fine-grained uncertainty relations of the state-independent form are derived for an arbitrary set of mutually unbiased bases. Such relations are extended with a recent notion of mutually unbiased measurements. The case of so-called mutually biased bases is considered in a similar manner. We also discuss a formulation of fine-grained uncertainty relations in the case of generalized measurements. The general approach is then applied to two measurements related to state discrimination. The case of three rank-one projective measurements is further examined in details. In particular, we consider fine-grained uncertainty relations for mutually unbiased bases in three-dimensional Hilbert space.
\end{abstract}

PACS numbers: 03.65.Ta, 03.67.-a

Keywords: uncertainty principle, spectral norm, state discrimination, mutually unbiased measurements, mutually biased bases

\section{INTRODUCTION}

The uncertainty principle [1] is one of fundamental limitations in description of the quantum world. Studies of such limitations are important in own rights as well as for applications in quantum information theory. Many quantitative formulations of the uncertainty principle were proposed. The Robertson formulation [2] gives a lower bound on product of the standard deviations of two quantum observables. The authors of [3] gave a reformulation dependent on the degree of Gaussianity of the state. The entropy-based approach is an important way to deal with quantum uncertainties [4 6]. Recently, noise-disturbance tradeoff relations have been formulated within the entropic approach [7]. Entropic uncertainty relations are applied in the security analysis of quantum key distribution, entanglement estimation, and quantum communication [8, 9]. Entropic uncertainty relations are reviewed in [10, 11].

In some respects, however, entropic approach is not considered as a complete formulation [12]. Indeed, each entropy is a function of probability distribution as a whole. Entropic bounds cannot distinguish the uncertainty inherent in obtaining a particular combination of the outcomes [12]. For these reasons, the so-called fine-grained uncertainty relations have been introduced [12]. Although entropic relations are quite appropriate in many questions, they are only a coarse way of describing quantum uncertainties. Fine-grained uncertainty relations for a one-qubit system were examined in [13]. Quantum uncertainties can also be expressed via various majorization relations, which are currently the subject of active research $[14[16]$.

The aim of the present work is to study fine-grained uncertainty relations for several measurements in arbitrary finite dimensions. The paper is organized as follows. In Section II the preliminary material is recalled. In Section III. we discuss fine-grained uncertainty relations in general. Explicit upper bounds are obtained for arbitrary set of mutually unbiased bases. We extend such bounds for the case of mutually unbiased measurements recently proposed in [17]. We also derive uncertainty bounds for mutually biased bases. In Section IV] we develop the proposed method with two measurements related to state discrimination. The case of three rank-one projectors is addressed in Section V It is exemplified with mutually unbiased bases in three-dimensional Hilbert space. In Section VI we conclude the paper with a summary of results.

\section{DEFINITIONS AND NOTATION}

In this section, preliminary facts are briefly outlined. Let $\mathcal{L}(\mathcal{H})$ be the space of linear operators on $d$-dimensional Hilbert space $\mathcal{H}$. By $\mathcal{L}_{\text {s.a. }}(\mathcal{H})$ and $\mathcal{L}_{+}(\mathcal{H})$, we will respectively mean the real space of Hermitian operators and the set of positive ones. For two operators $\mathrm{X}, \mathrm{Y} \in \mathcal{L}(\mathcal{H})$, their Hilbert-Schmidt product is defined as [18]

$$
\langle\mathrm{X}, \mathrm{Y}\rangle_{\mathrm{hs}}:=\operatorname{tr}\left(\mathrm{X}^{\dagger} \mathrm{Y}\right) \text {. }
$$

*Electronic address: alexrastegin@mail.ru 
This inner product induces the norm $\|X\|_{2}=\langle X, X\rangle_{\text {hs }}^{1 / 2}$. For any $X \in \mathcal{L}(\mathcal{H})$, we put $|X| \in \mathcal{L}_{+}(\mathcal{H})$ as a unique positive square root of $\mathbf{X}^{\dagger} \mathbf{X}$. The eigenvalues of $|\mathrm{X}|$ counted with multiplicities are the singular values of $\mathbf{X}$, written $\sigma_{j}(\mathbf{X})$. For all real $q \geq 1$, the Schatten $q$-norm is defined as [18]

$$
\|\mathrm{X}\|_{q}:=\left(\sum_{j=1}^{d} \sigma_{j}(\mathrm{X})^{q}\right)^{1 / q} .
$$

This family gives the trace norm $\|\mathrm{X}\|_{1}=\operatorname{tr}|\mathrm{X}|$ for $q=1$, the Hilbert-Schmidt norm $\|\mathrm{X}\|_{2}=\sqrt{\operatorname{tr}\left(\mathrm{X}^{\dagger} \mathrm{X}\right)}$ for $q=2$, and the spectral norm

$$
\|\mathbf{X}\|_{\infty}=\max \left\{\sigma_{j}(\mathrm{X}): 1 \leq j \leq d\right\}
$$

for $q=\infty$. Note that the norm (2) is merely the vector $q$-norm of the tuple of singular values of X. For any operator $\mathrm{A} \in \mathcal{L}_{+}(\mathcal{H})$, the spectral norm coincides with the maximum of its eigenvalues.

In general, quantum measurements are treated within the POVM formalism [19]. Let $\mathcal{M}=\left\{\mathrm{M}_{j}\right\}$ be a set of elements $\mathrm{M}_{j} \in \mathcal{L}_{+}(\mathcal{H})$, satisfying the completeness relation

$$
\sum_{j} \mathrm{M}_{j}=\mathbb{1}
$$

Here, the symbol $\mathbb{1}$ denotes the identity operator on $\mathcal{H}$. The set $\mathcal{M}=\left\{\mathrm{M}_{j}\right\}$ is a positive operator-valued measure $(\mathrm{POVM})$. Let the pre-measurement state of a system be described by density operator $\boldsymbol{\rho} \in \mathcal{L}_{+}(\mathcal{H})$ such $\operatorname{that} \operatorname{tr}(\boldsymbol{\rho})=1$. The probability of $j$-th outcome is then written as [19]

$$
p_{j}(\mathcal{M} \mid \boldsymbol{\rho})=\operatorname{tr}\left(\mathrm{M}_{j} \boldsymbol{\rho}\right) \text {. }
$$

When POVM elements are all orthogonal projectors, we deal with a projective measurement. Unlike projective measurements, a number of different outcomes in POVM measurements may exceed dimensionality of the Hilbert space [19]. For all normalized states $|\psi\rangle \in \mathcal{H}$, we have

$$
\left\langle\psi\left|\mathrm{M}_{j}\right| \psi\right\rangle \leq\left\|\mathrm{M}_{j}\right\|_{\infty} .
$$

This inequality is saturated with a corresponding eigenvector of $\mathrm{M}_{j}$. Combining (6) with the spectral decomposition of $\boldsymbol{\rho}$, we further obtain

$$
p_{j}(\mathcal{M} \mid \boldsymbol{\rho}) \leq\left\|\mathrm{M}_{j}\right\|_{\infty} .
$$

Irrespectively to the pre-measurement state, therefore, probabilities of the form (5) can generally be strictly less than 1. Of course, each probability of a projective measurement can reach the maximum 1. Discussion of entropic uncertainty relations for POVMs can be found in [20].

Some types of quantum measurements are of special interest in quantum information processing. Let $\mathcal{B}^{(1)}=\left\{\left|b_{j}^{(1)}\right\rangle\right\}$ and $\mathcal{B}^{(2)}=\left\{\left|b_{k}^{(2)}\right\rangle\right\}$ be two orthonormal bases in $d$-dimensional Hilbert space $\mathcal{H}$. When for all $j$ and $k$ we have

$$
\left|\left\langle b_{j}^{(1)} \mid b_{k}^{(2)}\right\rangle\right|=\frac{1}{\sqrt{d}}
$$

these bases are mutually unbiased [21]. The set $\mathbb{B}=\left\{\mathcal{B}^{(1)}, \ldots, \mathcal{B}^{(N)}\right\}$ is a set of mutually unbiased bases (MUBs), when any two bases from $\mathbb{B}$ are mutually unbiased. For example, three eigenbases of the Pauli matrices are mutually unbiased. Known constructions for MUBs have been considered in [22 25]. Various applications of MUBs in quantum theory are reviewed in [21]. It is known that MUBs for higher-dimensional orbital-angular-momentum states can be used to encode bits of information in alignment with the BB84 protocol [26 28]. Experimental studies of such propositions heve been addressed in [29, 30]. Entropic uncertainty relations for $d+1$ mutually unbiased bases in $d$-dimensional Hilbert space were derived in [31, 32]. The author of [32] also gave the exact bounds for the qubit case $d=2$. These exact relations have been generalized with use of the Tsallis and Rényi entropies [33, 34]. Specific uncertainty relations for MUBs and some applications were examined in [35, 36].

Basic constructions of MUBs are related to the case, when $d$ is power of a prime number. If the dimensionality is another composite number, maximal sets of MUBs are an open problem 21]. Hence, we may try to fit "unbiasedness" with weaker conditions. Recently, the authors of [17] proposed the concept of mutually unbiased measurements. Let us consider two POVM measurements $\mathcal{M}=\left\{\mathrm{M}_{j}\right\}$ and $\mathcal{N}=\left\{\mathrm{N}_{k}\right\}$. Each of them contains $d$ elements such that

$$
\begin{aligned}
& \operatorname{tr}\left(\mathrm{M}_{j}\right)=\operatorname{tr}\left(\mathrm{N}_{k}\right)=1, \\
& \operatorname{tr}\left(\mathrm{M}_{j} \mathrm{~N}_{k}\right)=\frac{1}{d} .
\end{aligned}
$$


The Hilbert-Schmidt product of two elements from the same POVM depends on the so-called efficiency parameter $\varkappa$ [17]. Namely, one has

$$
\operatorname{tr}\left(\mathrm{M}_{j} \mathrm{M}_{k}\right)=\delta_{j k} \varkappa+\left(1-\delta_{j k}\right) \frac{1-\varkappa}{d-1},
$$

and similarly for the elements of $\mathcal{N}$. The efficiency parameter satisfies [17]

$$
\frac{1}{d}<\varkappa \leq 1
$$

For $\varkappa=1 / d$ we have the trivial case, in which $\mathrm{M}_{j}=\mathbb{1} / d$ for all $j$. The value $\varkappa=1$, when possible, leads to the standard case of mutually unbiased bases. More precise bounds on $\varkappa$ will depend on a construction of measurement operators. The efficiency parameter shows how close the measurement operators are to rank-one projectors [17]. For the given $\varkappa$, we take the set $\mathbb{M}=\left\{\mathcal{M}^{(1)}, \ldots, \mathcal{M}^{(N)}\right\}$ of POVMs satisfying (11). When each two POVMs also obey conditions of the forms (9) and (10), the set $\mathbb{M}$ is a set of mutually unbiased measurements (MUMs). If we allow $\varkappa \neq 1$, then one can build $d+1$ MUMs in $d$-dimensional Hilbert space for arbitrary $d[17]$.

Mutually unbiased bases are used in multi-level schemes of quantum secret sharing [28]. So-called mutually biased bases form another important tool for constructing secret-sharing protocols [28]. By $|x\rangle$, we mean kets of the standard basis in $d$-dimensional Hilbert space. Let us begin with the unitary transformation

$$
\mathrm{V}_{F}|x\rangle=\frac{1}{\sqrt{d}} \sum_{y=0}^{d-1} \exp (\mathbf{i} x y \phi)|y\rangle
$$

where $\phi=2 \pi / d$ and $x \in\{0, \ldots, d-1\}$. In the computational basis, the Fourier transform acts as (13). To each $t \in\{0, \ldots, d-1\}$, we then assign the set of $d$ vectors [28]

$$
\left|a_{x}^{(t)}\right\rangle=\mathrm{V}_{F}|x\rangle+\frac{\exp (\mathrm{i} t \phi)-1}{\sqrt{d}}|0\rangle .
$$

The sets $\mathcal{A}^{(t)}=\left\{\left|a_{x}^{(t)}\right\rangle\right\}$ are all orthonormal and complete. Following [28], we call them mutually biased bases (MBBs). Simple calculations give the inner product

$$
\left\langle a_{x}^{(s)} \mid a_{y}^{(t)}\right\rangle=\delta_{x y}+\frac{\exp (\mathrm{i}(t-s) \phi)-1}{d} .
$$

Similarly to MUBs, mutually biased bases can be used in building effective schemes of quantum secret sharing [28]. In the next section, we will derive fine-grained uncertainty relations for MUBs, MUMs, and MBBs.

\section{ON FINE-GRAINED UNCERTAINTY RELATIONS IN GENERAL}

In this section, we consider the concept of fine-grained uncertainty relations in principle. A unified approach to their deriving is then discussed. Further, some general results with an arbitrary number of unbiased measurements are obtained. The case of $d$ MBBs is addressed as well. The notion of fine-grained uncertainty relations has been introduced in [12]. Some relations of such a kind for a one-qubit system were examined in [13].

In some respects, entropies give a coarse way of measuring uncertainty and incompatibility of a set of quantum measurements [12, 37]. This approach does not distinguish the uncertainty inherent in obtaining a concrete string of outcomes for different measurements. Hence, the authors of [12] proposed examining inequalities for particular sets of outcomes. An alternate approach to measure incompatibility of noncommuting observables was considered in [37].

Let us consider the corresponding formulation for a set of $N$ MUBs in $d$-dimensional space. In every base, we will number kets by integers from 0 up to $d-1$. To each $t=1, \ldots, N$, we assign an integer $j(t) \in\{0, \ldots, d-1\}$. Assuming equal weights, we introduce the quantity

$$
\max _{\boldsymbol{\rho}} \frac{1}{N} \sum_{t=1}^{N} p_{j(t)}\left(\mathcal{B}^{(t)} \mid \boldsymbol{\rho}\right)
$$

Suppose that we have obtained upper bounds on the sum (16) for all possible strings $j(1), \ldots, j(N)$. Such upper bounds will form a fine-grained uncertainty relation for a set of $N$ MUBs. In general, fine-grained uncertainty 
relations with unequal weights may be of interest [12]. Then upper bounds will also depend on chosen probabilities of different measurements.

We now consider formulation of fine-grained uncertainty relations in the case of $N$ generalized measurements. We should take into account the fact that particular probabilities may never reach the value 1 . The unambiguous state discrimination provides one example of such a kind. Particular probabilities are bounded from above according to (7). For a particular choice of POVM elements, one per each taken measurement, we herewith take summarized norms:

$$
S:=\sum_{t=1}^{N}\left\|\mathrm{M}_{j(t)}^{(t)}\right\|_{\infty} .
$$

To obtain fine-grained uncertainty relations, we aim to estimate from above all the possible quantities of the form

$$
\max _{\boldsymbol{\rho}} \frac{1}{S} \sum_{t=1}^{N} p_{j(t)}\left(\mathcal{M}^{(t)} \mid \boldsymbol{\rho}\right)
$$

In this approach, different measurements are assumed to be made with equal probabilities. Upper bounds on quantities of the form (18) will give a fine-grained uncertainty relation.

Let us consider fine-grained uncertainty relations for MUBs. The sum under maximization in (16) can be represented as $\operatorname{tr}(B \boldsymbol{\rho})$ in terms of the positive operator

$$
\mathrm{B}=\frac{1}{N} \sum_{t=1}^{N}\left|b_{j}^{(t)}\right\rangle\left\langle b_{j}^{(t)}\right|
$$

Here, each index $j$ is prescribed for the given $t$. A simple bound on the quantity (16) follows from the Cauchy-Schwarz inequality. Applying it to the Hilbert-Schmidt inner product, we obtain

$$
\langle\mathrm{B}, \boldsymbol{\rho}\rangle_{\mathrm{hs}} \leq\|\mathrm{B}\|_{2}\|\rho\|_{2}
$$

Due to (8), we further calculate the squared Hilbert-Schmidt norm

$$
\|\mathrm{B}\|_{2}^{2}=\operatorname{tr}\left(\mathrm{B}^{\dagger} \mathrm{B}\right)=\frac{1}{N^{2}}\left(N+\frac{N^{2}-N}{d}\right) .
$$

Combining this with (20) leads to a state-dependent bound

$$
\frac{1}{N} \sum_{t=1}^{N} p_{j(t)}\left(\mathcal{B}^{(t)} \mid \boldsymbol{\rho}\right) \leq \sqrt{\operatorname{tr}\left(\boldsymbol{\rho}^{2}\right)} \sqrt{\frac{N+d-1}{N d}}
$$

Following [38], we may regard (22) as a purity-based uncertainty bound. Purity-based entropic uncertainty relations were recently considered in [38]. For any pure state $|\psi\rangle \in \mathcal{H}$, we further write

$$
\frac{1}{N} \sum_{t=1}^{N} p_{j(t)}\left(\mathcal{B}^{(t)} \mid \psi\right) \leq \sqrt{\frac{N+d-1}{N d}} .
$$

This bound also gives a state-independent upper bound, i.e., a fine-grained uncertainty relation. It turns out that the uncertainty relation (23) can be improved. As state-independent formulations are of great interest in quantum information theory, we address this question in more details. Estimating the spectral norm of (19), the following result takes place.

Proposition 1 Let $\mathbb{B}=\left\{\mathcal{B}^{(1)}, \ldots, \mathcal{B}^{(N)}\right\}$ be a set of $M U B$ s in d-dimensional Hilbert space $\mathcal{H}$, and let some integer $j(t) \in\{0, \ldots, d-1\}$ be assigned to each $t=1, \ldots, N$. For arbitrary density matrix $\boldsymbol{\rho}$, we have

$$
\frac{1}{N} \sum_{t=1}^{N} p_{j(t)}\left(\mathcal{B}^{(t)} \mid \boldsymbol{\rho}\right) \leq \frac{1}{d}\left(1+\frac{d-1}{\sqrt{N}}\right) .
$$


Proof. Similarly to (7), we have $\operatorname{tr}(\mathrm{B} \rho) \leq\|\mathrm{B}\|_{\infty}$. In appendix $\mathrm{A}$ of the paper [39], we have shown the following. For any $d$-dimensional vector $w$, the corresponding vector norms satisfy

$$
\|w\|_{\infty} \leq \frac{1}{d}\left(\|w\|_{1}+\sqrt{d-1} \sqrt{d\|w\|_{2}^{2}-\|w\|_{1}^{2}}\right) .
$$

By construction, the operator (19) obeys $\|B\|_{1}=\operatorname{tr}(B)=1$. Combining this with (21), we write the relation

$$
d\|\mathrm{~B}\|_{2}^{2}-\|\mathrm{B}\|_{1}^{2}=\frac{d}{N}\left(1+\frac{N-1}{d}\right)-1=\frac{d-1}{N} .
$$

Applying (25) to the singular values of $B$, the norm $\|B\|_{\infty}$ is bounded from above by the right-hand side of (24).

The inequality (24) is a fine-grained uncertainty relation of the state-independent form for an arbitrary set of MUBs. By construction, this relation is approximate. For a small number of measurements, exact results may be obtained. Some of such cases are considered in the next sections. Let us compare the state-independent bounds (23) and (24). Some calculations show that the right-hand side of (24) cannot exceed (23). We now suppose that $d$ is a prime power and, herewith, there exists a set of $N=d+1$ MUBs. To compare the bounds (23) and (24), we may calculate a relative difference between them. For instance, this relative difference is about $6 \%$ for $d=3$, about $13 \%$ for $d=8$, and it increases with growth of dimensionality. For sufficiently large $d$, the relative difference tends to $\sqrt{2}-1$ that is about 40\%. In high dimensions, therefore, the state-independent bound (24) is significantly better. Thus, our method has allowed to improve essentially the obvious bound (23). The result (24) can easily be extended to mutually unbiased measurements. The corresponding relation is formulated as follows.

Proposition 2 Let $\mathbb{M}=\left\{\mathcal{M}^{(1)}, \ldots, \mathcal{M}^{(N)}\right\}$ be a set of mutually unbiased measurements of the efficiency $\varkappa$ in $d$ dimensional Hilbert space $\mathcal{H}$. Let an integer $j(t)$ be assigned to each $t=1, \ldots, N$. For arbitrary density matrix $\boldsymbol{\rho}$, we have

$$
\frac{1}{N} \sum_{t=1}^{N} p_{j(t)}\left(\mathcal{M}^{(t)} \mid \boldsymbol{\rho}\right) \leq \frac{1}{d}\left(1+\sqrt{\frac{(d-1)(\varkappa d-1)}{N}}\right) .
$$

Proof. We can proceed similarly to the previous proof. Instead of (19), we consider the positive operator

$$
\mathrm{Q}=\frac{1}{N} \sum_{t=1}^{N} \mathrm{M}_{j}^{(t)}
$$

Due to (9), we again have $\|Q\|_{1}=\operatorname{tr}(Q)=1$. Using (10) and (11), we further calculate the squared Hilbert-Schmidt norm

$$
\|\mathrm{Q}\|_{2}^{2}=\operatorname{tr}\left(\mathrm{Q}^{\dagger} \mathrm{Q}\right)=\frac{1}{N^{2}}\left(N \varkappa+\frac{N^{2}-N}{d}\right) .
$$

Corresponding substitutions immediately gives the upper bound (27).

The inequality (27) gives a fine-grained uncertainty relation for any set of MUMs. As only conditions of the forms (91) and (10) were used in the proof, the bound is independent of details of construction of measurement elements. In the case $\varkappa=1$, the result (27) is reduced to (24). With growth of the efficiency parameter $\varkappa$, the right-hand side of (27) increases from $1 / d$ up to the right-hand side of (24). Finally, we note that the Cauchy-Schwarz inequality leads to the state-dependent relation

$$
\frac{1}{N} \sum_{t=1}^{N} p_{j(t)}\left(\mathcal{M}^{(t)} \mid \boldsymbol{\rho}\right) \leq \sqrt{\operatorname{tr}\left(\boldsymbol{\rho}^{2}\right)} \sqrt{\frac{N+\varkappa d-1}{N d}} .
$$

Like (22), we merely use here an analog of (20) and (29). The inequality (30) provides a purity-based form of uncertainty bounds for any set of MUMs. For pure state $|\psi\rangle \in \mathcal{H}$, we obtain

$$
\frac{1}{N} \sum_{t=1}^{N} p_{j(t)}\left(\mathcal{M}^{(t)} \mid \psi\right) \leq \sqrt{\frac{N+\varkappa d-1}{N d}} .
$$

It is a state-independent bound, which takes place for all states. This state-independent bound is sufficiently weaker than (27). For instance, in the limiting case $\varkappa \rightarrow 1 / d$ we have the upper bounds $1 / d$ from (27) and only $\sqrt{1 / d}$ from (31). These two values considerably differ for large $d$.

In a similar manner, we will obtain a fine-grained uncertainty relation for $d$ MBBs in $d$-dimensional Hilbert space. Recall that such bases are used in some schemes of quantum secret sharing [28]. We have the following statement. 
Proposition 3 Let $\mathbb{A}=\left\{\mathcal{A}^{(0)}, \ldots, \mathcal{A}^{(d-1)}\right\}$ be the set of $d$ MBBs in d-dimensional Hilbert space $\mathcal{H}$. Let the given integers $x(0), \ldots, x(d-1)$ form a permutation of the integers $0, \ldots, d-1$. For arbitrary density matrix $\boldsymbol{\rho}$, we have

$$
\frac{1}{d} \sum_{t=0}^{d-1} p_{x(t)}\left(\mathcal{A}^{(t)} \mid \boldsymbol{\rho}\right) \leq \frac{1}{d}\left(1+\sqrt{2-\frac{2}{d}}\right) .
$$

Proof. Let us consider the positive operator

$$
\mathrm{A}=\frac{1}{d} \sum_{t=0}^{d-1}\left|a_{x}^{(t)}\right\rangle\left\langle a_{x}^{(t)}\right| .
$$

Here, we have $\|\mathrm{A}\|_{1}=\operatorname{tr}(\mathrm{A})=1$. The precondition of the theorem implies $x(s) \neq x(t)$ for $s \neq t$. Combining this with (15) then gives

$$
d^{2}\|\mathrm{~A}\|_{2}^{2}=d+\frac{1}{d^{2}} \sum_{\substack{s, t=0 \\ s \neq t}}^{d-1}|\exp (\mathrm{i}(t-s) \phi)-1|^{2},
$$

where $\phi=2 \pi / d$. Note that the condition $s \neq t$ can quite be ignored in the sum of the right-hand side of (34). Hence, this sum is calculated as

$$
2 d^{2}-2 \sum_{s, t=0}^{d-1} \cos ((t-s) \phi)=2 d^{2} .
$$

Indeed, the sum of cosines can be rewritten as the real part of the sum

$$
\sum_{s=0}^{d-1} \exp (-\mathrm{i} s \phi) \sum_{t=0}^{d-1} \exp (\mathrm{i} t \phi)=0 .
$$

Therefore, we have

$$
d\|\mathrm{~A}\|_{2}^{2}-\|\mathrm{A}\|_{1}^{2}=\frac{2}{d} .
$$

Using (25), the spectral norm $\|\mathrm{A}\|_{\infty}$ is bounded from above by the right-hand side of (32).

The inequality (32) gives a fine-grained uncertainty relation of the set of $d$ MBBs. It is instructive to compare this bound with the upper bound (24) taken with $N=d$ MUBs. Except for low dimensions, the bound (24) is larger enough. For large $d$, the bound (32) is approximately $(\sqrt{2}+1) / d$, whereas the bound (24) with $N=d$ is approximately $\sqrt{1 / d}$. This observation is a manifestation of the fact that mutually biased bases somehow deviate from an equipartition provided by mutually unbiased bases.

\section{RELATIONS FOR TWO MEASUREMENTS USED IN STATE DISCRIMINATION}

In this section, we consider fine-grained uncertainty relations for two measurements related to state discrimination. Discrimination of quantum states is important in applications [40, 41] and leading to interesting problems [42]. There exist two basic approaches to discrimination between non-identical pure states $\left|\theta_{+}\right\rangle$and $\left|\theta_{-}\right\rangle$, which we will parameterize as

$$
\left|\theta_{+}\right\rangle=\left(\begin{array}{c}
\cos \theta \\
\sin \theta
\end{array}\right), \quad\left|\theta_{-}\right\rangle=\left(\begin{array}{c}
\cos \theta \\
-\sin \theta
\end{array}\right)
$$

As the inner product is $\left\langle\theta_{+} \mid \theta_{-}\right\rangle=\cos 2 \theta$, we focus our consideration to the values $2 \theta \in(0 ; \pi / 2)$ and, herewith, to non-identical and non-orthogonal states. Therefore, the value $\theta=0$ should be left out. In the Helstrom scheme [40], the optimal measurement is reached by the set $\mathcal{N}=\left\{\mathrm{N}_{+}, \mathrm{N}_{-}\right\}$of two projectors

$$
\mathrm{N}_{ \pm}=\left|n_{ \pm}\right\rangle\left\langle n_{ \pm}|, \quad| n_{ \pm}\right\rangle=\frac{1}{\sqrt{2}}\left(\begin{array}{c}
1 \\
\pm 1
\end{array}\right)
$$


In the Helstrom scheme of distinguishing between $\left|\theta_{+}\right\rangle$and $\left|\theta_{-}\right\rangle$, the average probability of correct answer is optimized [40]. For equiprobable states, this probability is equal to

$$
P_{D}=\frac{1}{2}\left(1+\sqrt{1-\left|\left\langle\theta_{+} \mid \theta_{-}\right\rangle\right|^{2}}\right)=\frac{1+\sin 2 \theta}{2} .
$$

Of course, the probability (40) becomes maximal for two orthogonal states.

The second approach known as the unambiguous discrimination is essential in quantum cryptography [43]. This approach sometimes gives an inconclusive answer, but never makes an error of mis-identification. Such a formulation is purely quantum in character. The generalized measurement, minimizing the probability of inconclusive answer, has been built in the papers [44 46]. In the context of quantum cryptography, however, another measurement for state discrimination may be more appropriate [47]. In the B92 scheme [48], the sender encodes bits 0 and 1 into nonorthogonal states $\left|\theta_{+}\right\rangle$and $\left|\theta_{-}\right\rangle$respectively. To implement quantum key distribution, the receiver must distinguish between $\left|\theta_{+}\right\rangle$and $\left|\theta_{-}\right\rangle$without an error of mis-identification. Of course, some bits are assumed to be lost. Let $\left|m_{j}\right\rangle$ be vector orthogonal to $\left|\theta_{j}\right\rangle$, i.e., $\left\langle m_{j} \mid \theta_{j}\right\rangle=0$ for $j= \pm$. In the basis $\{|0\rangle,|1\rangle\}$, we have

$$
\left|m_{+}\right\rangle=\left(\begin{array}{c}
\sin \theta \\
-\cos \theta
\end{array}\right), \quad\left|m_{-}\right\rangle=\left(\begin{array}{c}
\sin \theta \\
\cos \theta
\end{array}\right) .
$$

In the original version [48], the receiver randomly measures one of two projectors $\left|m_{+}\right\rangle\left\langle m_{+}\right|$and $\left|m_{-}\right\rangle\left\langle m_{-}\right|$. More economical scheme is described by the three-element POVM $\mathcal{M}=\left\{\mathrm{M}_{+}, \mathrm{M}_{-}, \mathrm{M}_{\text {? }}\right\}$, where

$$
\begin{aligned}
\mathrm{M}_{+} & =\frac{1}{1+\cos 2 \theta}\left|m_{-}\right\rangle\left\langle m_{-}\left|, \quad \mathrm{M}_{-}=\frac{1}{1+\cos 2 \theta}\right| m_{+}\right\rangle\left\langle m_{+}\right|, \\
\mathrm{M}_{?} & =\mathbb{1}-\mathrm{M}_{+}-\mathrm{M}_{-} .
\end{aligned}
$$

By construction, we have $\left\langle\theta_{-}\left|\mathrm{M}_{+}\right| \theta_{-}\right\rangle=\left\langle\theta_{+}\left|\mathrm{M}_{-}\right| \theta_{+}\right\rangle=0$. If the POVM measurement $\mathcal{M}$ has given the outcome "+", then the input of discrimination process was certainly $\left|\theta_{+}\right\rangle$; the outcome "-" implies that the input was $\left|\theta_{-}\right\rangle$. Note that third inconclusive outcome "?" is allowed, though the problem is effectively two-dimensional. For the parametrization (38), the operator $\mathrm{M}_{\text {? }}$ is represented as

$$
\mathrm{M}_{?}=\frac{2 \cos 2 \theta}{1+\cos 2 \theta}|0\rangle\langle 0| \text {. }
$$

This operator becomes zero with $2 \theta=\pi / 2$, when the states (38) are orthogonal. For equiprobable states $\left|\theta_{ \pm}\right\rangle$, the average probability of conclusive answer reads

$$
\frac{(\sin 2 \theta)^{2}}{1+\cos 2 \theta}=1-\left|\left\langle\theta_{+} \mid \theta_{-}\right\rangle\right|
$$

This probability vanishes for parallel states and reaches 1 for two orthogonal states.

For brevity, we denote the overlap between states $\left|\theta_{ \pm}\right\rangle$by $\eta$, i.e., $\eta=\cos 2 \theta$. Let us consider matrices

$$
\mathrm{N}_{ \pm}+\mathrm{M}_{?}=\frac{1}{2}\left(\begin{array}{cc}
1+2 a(\eta) & \pm 1 \\
\pm 1 & 1
\end{array}\right), \quad a(\eta):=\frac{2 \eta}{1+\eta}
$$

By usual calculations, we then obtain

$$
\left\|\mathrm{N}_{ \pm}+\mathrm{M}_{?}\right\|_{\infty}=\frac{1}{2}\left(1+a(\eta)+\sqrt{1+a(\eta)^{2}}\right)
$$

This value can be reached with the corresponding eigenvectors of the matrices $\mathrm{N}_{ \pm}+\mathrm{M}_{?}$. Using $\left\|\mathrm{M}_{?}\right\|_{\infty}=a(\eta)$, the exact uncertainty bound is written as

$$
\max _{\boldsymbol{\rho}} \frac{p_{ \pm}(\mathcal{N} \mid \boldsymbol{\rho})+p_{?}(\mathcal{M} \mid \boldsymbol{\rho})}{1+\left\|\mathrm{M}_{?}\right\|_{\infty}}=\frac{1}{2}\left(1+\frac{\sqrt{1+2 \eta+5 \eta^{2}}}{1+3 \eta}\right)
$$

The right-hand side of (47) is the upper bound on rescaled sum of two particular probabilities. This bound decreases from the value 1 for $\eta=0$ (orthogonal states) up to the infimum $(2+\sqrt{2}) / 4 \approx 0.854$ for $\eta \rightarrow 1^{-}$(parallel states). 
We will now consider the matrices $\mathrm{N}_{+}+\mathrm{M}_{+}$and $\mathrm{N}_{-}+\mathrm{M}_{-}$. In the chosen basis, they are respectively written as

$$
\frac{1}{2(1+\eta)}\left(\begin{array}{cc}
2 & \pm(1+\eta+\sin 2 \theta) \\
\pm(1+\eta+\sin 2 \theta) & 2(1+\eta)
\end{array}\right)
$$

Calculating eigenvalues, we then obtain

$$
\left\|\mathrm{N}_{+}+\mathrm{M}_{+}\right\|_{\infty}=\left\|\mathrm{N}_{-}+\mathrm{M}_{-}\right\|_{\infty}=\frac{2+\eta+\sqrt{\eta^{2}+4(1+\eta) P_{D}}}{2(1+\eta)} .
$$

Recall that $P_{D}$ denotes the right-hand side of (40). Combining (49) with $\left\|\mathrm{M}_{ \pm}\right\|_{\infty}=(1+\eta)^{-1}$ gives the exact uncertainty bound

$$
\max _{\boldsymbol{\rho}} \frac{p_{j}(\mathcal{N} \mid \boldsymbol{\rho})+p_{j}(\mathcal{M} \mid \boldsymbol{\rho})}{1+\left\|\mathrm{M}_{j}\right\|_{\infty}}=\frac{1}{2}\left(1+\frac{\sqrt{\eta^{2}+4(1+\eta) P_{D}}}{2+\eta}\right),
$$

where $j= \pm$. The bound (50) decreases from the value 1 for $\eta=0$ (orthogonal states) up to the infimum $1 / 2+\sqrt{5} / 6 \approx$ 0.873 for $\eta \rightarrow 1^{-}$(parallel states).

Finally, we consider the matrices $\mathrm{N}_{+}+\mathrm{M}_{-}$and $\mathrm{N}_{-}+\mathrm{M}_{+}$. These matrices are respectively expressed as

$$
\frac{1}{2(1+\eta)}\left(\begin{array}{cc}
2 & \pm(1+\eta-\sin 2 \theta) \\
\pm(1+\eta-\sin 2 \theta) & 2(1+\eta)
\end{array}\right)
$$

By calculations, we further obtain

$$
\left\|\mathrm{N}_{+}+\mathrm{M}_{-}\right\|_{\infty}=\left\|\mathrm{N}_{-}+\mathrm{M}_{+}\right\|_{\infty}=\frac{2+\eta+\sqrt{\eta^{2}+4(1+\eta) P_{E}}}{2(1+\eta)} .
$$

Here, the term $P_{E}=1-P_{D}$ is the error probability in the Helstrom scheme. The exact uncertainty bound reads

$$
\max _{\boldsymbol{\rho}} \frac{p_{j}(\mathcal{N} \mid \boldsymbol{\rho})+p_{k}(\mathcal{M} \mid \boldsymbol{\rho})}{1+\left\|\mathrm{M}_{k}\right\|_{\infty}}=\frac{1}{2}\left(1+\frac{\sqrt{\eta^{2}+4(1+\eta) P_{E}}}{2+\eta}\right),
$$

where the pair $(j, k)$ is either $(+,-)$ or $(-,+)$. Unlike (50), the bound (53) increases with growing $\eta$. The bound (53) is equal to $1 / 2$ for $\eta=0$ (orthogonal states) and tends to $1 / 2+\sqrt{5} / 6 \approx 0.873$ in the limit $\eta \rightarrow 1^{-}$(parallel states). Note also that the quantities (50) and (53) coincide in this limit. It is a manifestation of the fact that states become indistinguishable. On the other hand, in the case of orthogonal states the quantity (50) twice exceeds (53). In combination, the formulas (47), (50), and (53) give an exact fine-grained uncertainty relation for two measurements used in quantum state discrimination.

\section{RELATIONS FOR THREE RANK-ONE PROJECTORS IN $d$-DIMENSIONAL SPACE}

In this section, we will derive fine-grained uncertainty relations for three MUBs in a finite-dimensional Hilbert space. The case of two rank-one projectors is effectively two-dimensional. Three projectors generally lead to a situation that cannot be reduced in such a way. We will also study a concrete example of certain physical interest. Our approach is based on calculation of the maximal eigenvalue of a certain positive matrix. This matrix is expressed as the sum of three rank-one projectors. Let $\left|b_{1}\right\rangle,\left|b_{2}\right\rangle$, and $\left|b_{3}\right\rangle$ be three unit vectors such that pairwise overlaps are all equal to $\eta<1$, i.e., $\left|\left\langle b_{j} \mid b_{k}\right\rangle\right|=\eta$ for $j \neq k$. We aim to find the spectral norm of the positive operator

$$
\left|b_{1}\right\rangle\left\langle b_{1}|+| b_{2}\right\rangle\left\langle b_{2}|+| b_{3}\right\rangle\left\langle b_{3}\right| \text {. }
$$

When the vectors are taken from the MUBs $\mathcal{B}^{(1)}, \mathcal{B}^{(2)}, \mathcal{B}^{(3)}$, i.e., $\left|b_{j}\right\rangle \in \mathcal{B}^{(j)}$, we have $\eta=d^{-1 / 2}$. We will assume that the subspace $\operatorname{span}\left\{\left|b_{1}\right\rangle,\left|b_{2}\right\rangle,\left|b_{3}\right\rangle\right\}$ is three-dimensional, though our approach is quite applicable in two actual dimensions. Of course, the two-dimensional case is easier to analyze.

We begin with three vectors, for which inner products are all real. First, we introduce a useful parametrization of involved vectors. Let us consider the sub-normalized vectors

$$
\begin{aligned}
\left|b_{1}^{\perp}\right\rangle & :=\left|b_{1}\right\rangle-\left|b_{3}\right\rangle\left\langle b_{3} \mid b_{1}\right\rangle, \\
\left|b_{2}^{\perp}\right\rangle & :=\left|b_{2}\right\rangle-\left|b_{3}\right\rangle\left\langle b_{3} \mid b_{2}\right\rangle .
\end{aligned}
$$


In the case considered, the inner product $\left\langle b_{1}^{\perp} \mid b_{2}^{\perp}\right\rangle$ can be assumed to be positive real. In the subspace $\operatorname{span}\left\{\left|b_{1}^{\perp}\right\rangle,\left|b_{2}^{\perp}\right\rangle\right\}$, we take orthonormal basis such that the first ket is proportional to $\left|b_{1}^{\perp}\right\rangle+\left|b_{2}^{\perp}\right\rangle$ and the second ket is proportional to $\left|b_{2}^{\perp}\right\rangle-\left|b_{1}^{\perp}\right\rangle$. The third auxiliary ket is merely equal to $\left|b_{3}\right\rangle$. Due to the defined auxiliary kets, we will deal with the following vectors:

$$
\left|c_{1}\right\rangle=\left(\begin{array}{c}
\alpha \\
-\beta \\
\eta
\end{array}\right), \quad\left|c_{2}\right\rangle=\left(\begin{array}{l}
\alpha \\
\beta \\
\eta
\end{array}\right), \quad\left|c_{3}\right\rangle=\left(\begin{array}{l}
0 \\
0 \\
1
\end{array}\right) .
$$

For the given $\eta$, the positive real numbers $\alpha$ and $\beta$ satisfy

$$
\begin{aligned}
& \alpha^{2}+\beta^{2}+\eta^{2}=1, \\
& \alpha^{2}-\beta^{2}+\eta^{2}=\eta .
\end{aligned}
$$

After calculations, we obtain

$$
\alpha=\sqrt{\frac{1+\eta-2 \eta^{2}}{2}}, \quad \beta=\sqrt{\frac{1-\eta}{2}} .
$$

The sum of three projectors then reads

$$
\left|c_{1}\right\rangle\left\langle c_{1}|+| c_{2}\right\rangle\left\langle c_{2}|+| c_{3}\right\rangle\left\langle c_{3}\right|=\left(\begin{array}{ccc}
2 \alpha^{2} & 0 & 2 \alpha \eta \\
0 & 2 \beta^{2} & 0 \\
2 \alpha \eta & 0 & 2 \eta^{2}+1
\end{array}\right) .
$$

The characteristic equation of the matrix (61) is written as

$$
\left(\lambda-2 \beta^{2}\right)\left(\lambda^{2}-(2+\eta) \lambda+2 \alpha^{2}\right)=0 .
$$

By calculations, we finally obtain the eigenvalue $1-\eta$ with multiplicity 2 and the eigenvalue $1+2 \eta$. Thus, the spectral norm of (61) is equal to $1+2 \eta$. Hence, we write an exact uncertainty bound

$$
\max _{\boldsymbol{\rho}} \frac{1}{3} \sum_{j=1}^{3}\left\langle c_{j}|\boldsymbol{\rho}| c_{j}\right\rangle=\frac{1+2 \eta}{3} .
$$

This relation is tight in the following sense. It is always saturated with the corresponding eigenvector of (61), when $\boldsymbol{\rho}=|\psi\rangle\langle\psi|$ and

$$
|\psi\rangle=\frac{1}{\sqrt{3(1+2 \eta)}}\left(\begin{array}{c}
\sqrt{2\left(1+\eta-2 \eta^{2}\right)} \\
0 \\
1+2 \eta
\end{array}\right) .
$$

This form is related to those basis that is used in the representation (57). The state (64) has the same overlap with each $\left|c_{j}\right\rangle$. This overlap is equal to the squared root of the right-hand side of (63). The right-hand side of (63) increases from the value $1 / 3$ for $\eta=0$ up to the value 1 for $\eta=1$. The former corresponds to three mutually orthogonal projectors, whereas the latter actually deals with one and the same projector.

We now consider a more complicated example. It is related to the four MUBs in three-dimensional Hilbert space. In principle, we may build some parametrization similarly to (57). It will include an additional phase factor. With explicitly given vectors, however, direct calculations are more appropriate. By $\omega=\exp (i 2 \pi / 3)$, we mean a primitive root of the unit. The normalized vectors of these bases are written as

$$
\begin{array}{lll}
\left\{\left(\begin{array}{l}
1 \\
0 \\
0
\end{array}\right),\left(\begin{array}{l}
0 \\
1 \\
0
\end{array}\right),\left(\begin{array}{l}
0 \\
0 \\
1
\end{array}\right)\right\}, & \left\{\frac{1}{\sqrt{3}}\left(\begin{array}{l}
1 \\
1 \\
1
\end{array}\right), \frac{1}{\sqrt{3}}\left(\begin{array}{c}
1 \\
\omega^{*} \\
\omega
\end{array}\right), \frac{1}{\sqrt{3}}\left(\begin{array}{c}
1 \\
\omega \\
\omega^{*}
\end{array}\right)\right\}, \\
\left\{\frac{1}{\sqrt{3}}\left(\begin{array}{l}
1 \\
\omega \\
1
\end{array}\right), \frac{1}{\sqrt{3}}\left(\begin{array}{l}
1 \\
1 \\
\omega
\end{array}\right), \frac{1}{\sqrt{3}}\left(\begin{array}{c}
\omega \\
1 \\
1
\end{array}\right)\right\}, & \left\{\frac{1}{\sqrt{3}}\left(\begin{array}{c}
1 \\
1 \\
\omega^{*}
\end{array}\right), \frac{1}{\sqrt{3}}\left(\begin{array}{c}
\omega^{*} \\
1 \\
1
\end{array}\right), \frac{1}{\sqrt{3}}\left(\begin{array}{c}
1 \\
\omega^{*} \\
1
\end{array}\right)\right\} .
\end{array}
$$

Here, the symbol $*$ denotes complex conjugation. When one of MUBs is taken as the standard base, other MUBs can be described in terms of complex Hadamard matrices. This fact has been used for classification of MUBs in low dimensions [49]. 
The four MUBs (65) - (66) give eigenbases of the four operators from the Weyl-Heisenberg group. They were applied in studies of complementarity of spin-1 observables [50]. These bases were also used in experimental study of higher-dimensional quantum key distribution protocols based on mutually unbiased bases [30]. The two bases (65) are respectively the eigenbases of the generalized Pauli operators

$$
Z=\left(\begin{array}{ccc}
1 & 0 & 0 \\
0 & \omega & 0 \\
0 & 0 & \omega^{*}
\end{array}\right), \quad X=\left(\begin{array}{lll}
0 & 0 & 1 \\
1 & 0 & 0 \\
0 & 1 & 0
\end{array}\right)
$$

Further, the two bases (66) are the eigenbases of the operators $Z \mathbf{Z X}$ and $\mathbf{Z} \mathbf{X}^{2}$. The vectors in each base are arranged according to the order of eigenvalues $1, \omega, \omega^{*}$. Except for own eigenbasis, the action of each of the four operators $Z$, $X, Z X, X^{2}$ leads to cyclic permutations in other three bases, sometimes with additional phase factors.

We consider fine-grained uncertainty relations for the projectors $\left|b_{1}\right\rangle\left\langle b_{1}|,| b_{2}\right\rangle\left\langle b_{2}\right|$, and $\left|b_{3}\right\rangle\left\langle b_{3}\right|$, where the used vectors are separately taken from three of the above bases (65)-666). Of course, no two vectors are taken from one and the same base. First, we consider the vectors

$$
\left|b_{1}\right\rangle=\frac{1}{\sqrt{3}}\left(\begin{array}{l}
1 \\
1 \\
1
\end{array}\right), \quad\left|b_{2}\right\rangle=\frac{1}{\sqrt{3}}\left(\begin{array}{l}
1 \\
\omega \\
1
\end{array}\right), \quad\left|b_{3}\right\rangle=\frac{1}{\sqrt{3}}\left(\begin{array}{c}
1 \\
\omega^{*} \\
1
\end{array}\right) .
$$

We easily calculate the sum of projectors

$$
\left|b_{1}\right\rangle\left\langle b_{1}|+| b_{2}\right\rangle\left\langle b_{2}|+| b_{3}\right\rangle\left\langle b_{3}\right|=\left(\begin{array}{ccc}
1 & 0 & 1 \\
0 & 1 & 0 \\
1 & 0 & 1
\end{array}\right) .
$$

This matrix has the trace 3 and the determinant 0 . The sum of second-order principal minors is equal to 2 . So, the characteristic equation is written as

$$
\lambda^{3}-3 \lambda^{2}+2 \lambda=0,
$$

whence the eigenvalues $0,1,2$ are found. Thus, we obtain the upper bound

$$
\max _{\boldsymbol{\rho}} \frac{1}{3} \sum_{j=1}^{3}\left\langle b_{j}|\rho| b_{j}\right\rangle=\frac{2}{3} .
$$

It is always saturated with the corresponding eigenvector of the matrix (69), when $\boldsymbol{\rho}=|\psi\rangle\langle\psi|$ and

$$
|\psi\rangle=\frac{1}{\sqrt{2}}\left(\begin{array}{l}
1 \\
0 \\
1
\end{array}\right)
$$

The relation (71) also holds for some other three-vector combinations such that no vectors are taken from one and the same base. For other combinations, the maximizing vector will differ from (72). Of course, it can easily be calculated with each concrete choice of three vectors.

Second, we consider the following unit vectors:

$$
\left|a_{1}\right\rangle=\left(\begin{array}{l}
0 \\
0 \\
1
\end{array}\right), \quad\left|a_{2}\right\rangle=\frac{1}{\sqrt{3}}\left(\begin{array}{l}
1 \\
\omega \\
1
\end{array}\right), \quad\left|a_{3}\right\rangle=\frac{1}{\sqrt{3}}\left(\begin{array}{c}
1 \\
\omega^{*} \\
1
\end{array}\right) .
$$

We should calculate the spectral norm of the matrix

$$
\left|a_{1}\right\rangle\left\langle a_{1}|+| a_{2}\right\rangle\left\langle a_{2}|+| a_{3}\right\rangle\left\langle a_{3}\right|=\frac{1}{3}\left(\begin{array}{ccc}
2 & -1 & 2 \\
-1 & 2 & -1 \\
2 & -1 & 5
\end{array}\right) .
$$

This matrix has the trace 3 and the determinant $1 / 3$. The sum of second-order principal minors is equal to 2 . Then the characteristic equation reads

$$
\lambda^{3}-3 \lambda^{2}+2 \lambda-\frac{1}{3}=0
$$


Solving this equation, we find the maximal eigenvalue

$$
\max \lambda=1+\frac{2}{\sqrt{3}} \cos \frac{\pi}{18} \approx 2.137 .
$$

Thus, we obtain an upper bound on the sum of three probabilities, namely

$$
\max _{\boldsymbol{\rho}} \frac{1}{3} \sum_{j=1}^{3}\left\langle a_{j}|\boldsymbol{\rho}| a_{j}\right\rangle=\frac{1}{3}\left(1+\frac{2}{\sqrt{3}} \cos \frac{\pi}{18}\right) \approx \frac{2.137}{3} .
$$

Some inspection shows that each combination of three unit vectors from a triple of MUBs of the set (65)-(66) gives either (70) or (75). The former corresponds to the determinant 0 , the latter corresponds to the determinant $1 / 3$. Thus, the relations (71) and (77) together give a fine-grained uncertainty relation for the above MUBs in 3-dimensional Hilbert space. Both the upper bounds (71) or (77) are strictly less than 1 . They reflect a complementarity of spin-1 observables. Using the quantities (71) and (77), we may estimate a quality of the bound of Proposition 1. Substituting $d=N=3$ into the right-hand side of (24), we obtain the number $(1+2 / \sqrt{3}) / 3 \approx 2.155 / 3$. It is close to (71) and very close to (77) from above. This gives an evidence for the fact that the approximate bound (24) is good enough, at least for low dimensions.

As was shown in [49], triples of MUBs in $d=3$ are all equivalent in the sense of several kinds of transformations applied to a string of Hadamard matrices. The set of used transformations includes an overall unitary matrix applied from the left, diagonal unitary matrices applied from the right, permutations of vectors within each basis, pairwise exchanges of two bases, and an overall complex conjugation. It can be checked that such operations will again lead to either (71) or (77). For example, multiplication by a diagonal unitary matrix from the right merely attaches phase factors to each column of the transformed matrix [49]. Thus, these bounds cover all fine-grained uncertainty relations for triplets of MUBs in the case $d=3$. The above method could be applied to triples of MUBs in other dimensions, but a situation becomes more complicated. For instance, the authors of [49] found a three-parameter family of triples in dimension four and two inequivalent triples in dimension five.

\section{CONCLUSION}

We have examined fine-grained uncertainty relations in the case of several quantum measurements. Formulation of fine-grained uncertainty relations for generalized measurements is first considered. Our approach is based on evaluation of spectral norms of the corresponding positive matrices. In discussed examples, we assume that different measurements have equal weights. However, the considered approach is also appropriate for cases, when measurements of interest have different probabilities. Explicit upper bounds are obtained for arbitrary set of mutually unbiased bases and then extended for the case of mutually unbiased measurements. In a similar manner, we considered uncertainty relations for so-called mutually biased bases essential in some schemes of quantum secret sharing. To illustrate an approach to generalized measurements, we analyzed a fine-grained uncertainty relation for two measurements used in state discrimination. One of them known as the unambiguous state discrimination is closely related to the B92 protocol of quantum cryptography. We also examined fine-grained uncertainty relations for three rank-one projective measurements. As an example, we further addressed the case of mutually unbiased bases in three-dimensional Hilbert space. Considered fine-grained uncertainty relations may be useful in studying secret-sharing and quantum-keydistribution protocols based on mutually biased or unbiased bases.

[1] Heisenberg, W.: Über den anschaulichen Inhalt der quantentheoretischen Kinematik und Mechanik. Zeitschrift für Physik 43, 172-198 (1927)

[2] Robertson, H.P.: The uncertainty principle. Phys. Rev. 34, 163-164 (1929)

[3] Mandilara, A., Cerf, N.J.: Quantum uncertainty relation saturated by the eigenstates of the harmonic oscillator. Phys. Rev. A 86, 030102(R) (2012)

[4] Białynicki-Birula, I., Mycielski, J.: Uncertainty relations for information entropy in wave mechanics. Commun. Math. Phys. 44, 129-132 (1975)

[5] Deutsch, D.: Uncertainty in quantum measurements. Phys. Rev. Lett. 50, 631-633 (1983)

[6] Maassen, H., Uffink, J.B.M.: Generalized entropic uncertainty relations. Phys. Rev. Lett. 60, 1103-1106 (1988)

[7] Buscemi, F., Hall, M.J.W., Ozawa, M., Wilde, M.M.: Noise and disturbance in quantum measurements: an informationtheoretic approach. Phys. Rev. Lett. 112, 050401 (2014) 
[8] Ng, H.Y.N., Berta, M., Wehner, S.: Min-entropy uncertainty relation for finite-size cryptography. Phys. Rev. A 86, 042315 (2012)

[9] Coles, P.J., Piani, M.: Improved entropic uncertainty relations and information exclusion relations. Phys. Rev. A 89, $022112(2014)$

[10] Wehner, S., Winter, A.: Entropic uncertainty relations - a survey. New J. Phys. 12, 025009 (2010)

[11] Białynicki-Birula, I., Rudnicki, Ł.: Entropic uncertainty relations in quantum physics. In Sen, K.D. (ed.): Statistical Complexity, 1-34. Springer, Berlin (2011)

[12] Oppenheim, J., Wehner, S.: The uncertainty principle determines the nonlocality of quantum mechanics. Science 330, 1072-1074 (2010)

[13] Ren, L.-H., Fan, H.: The fine-grained uncertainty relation for mutually unbiased bases. arXiv:1312.6744 [quant-ph] (2013)

[14] Friedland, S., Gheorghiu, V., Gour, G.: Universal uncertainty relations, Phys. Rev. Lett. 111, 230401 (2013)

[15] Puchała, Z., Rudnicki, Ł., Życzkowski, K.: Majorization entropic uncertainty relations. J. Phys. A: Math. Theor. 46, $272002(2013)$

[16] Rudnicki, Ł., Puchała, Z., Życzkowski, K.: Strong majorization entropic uncertainty relations. Phys. Rev. A 89, 052115 (2014)

[17] Kalev, A., Gour, G.: Mutually unbiased measurements in finite dimensions. New J. Phys. 16, 053038 (2014)

[18] Watrous, J.: Theory of Quantum Information. (University of Waterloo: 2011) http://www.cs.uwaterloo.ca/ watrous/CS766/

[19] Peres, A.: Quantum Theory: Concepts and Methods. (Dordrecht: Kluwer, 1993)

[20] Coles, P.J., Colbeck, R., Yu, L., Zwolak, M.: Uncertainty relations from simple entropic properties. Phys. Rev. Lett. 108, 210405 (2012)

[21] Durt, T. Englert, B.-G., Bengtsson, I., Życzkowski, K.: On mutually unbiased bases. Int. J. Quantum Inf. 8, 535-640 (2010)

[22] Wootters, W.K., Fields, B.D.: Optimal state-determination by mutually unbiased measurements. Ann. Phys. 191, 363-381 (1989)

[23] Bandyopadhyay, S., Boykin, P.O., Roychowdhury, V., Vatan, F.: A new proof for the existence of mutually unbiased bases. Algorithmica 34, 512-528 (2002)

[24] Klappenecker, A., Röttler, M.: Constructions of mutually unbiased bases. In: Finite Fields and Applications, Lecture Notes in Computer Science, vol. 2948, 137-144. Springer, Berlin (2004)

[25] Wocjan, P., Beth, T.: New construction of mutually unbiased bases in square dimensions. Quantum Inf. Comput. 5, 93-101 (2005)

[26] Bechmann-Pasquinucci, H., Peres, A.: Quantum cryptography with 3-state systems. Phys. Rev. Lett. 85, 3313 (2000)

[27] Cerf, N.J., Bourennane, M., Karlsson, A.,, Gisin, N.: Security of quantum key distribution using $d$-level systems. Phys. Rev. Lett. 88, 127902 (2002)

[28] Yu, I.-C., Lin, F.-L., Huang, C.-Y.: Quantum secret sharing with multilevel mutually (un-)biased bases. Phys. Rev. A 78, $012344(2008)$

[29] Gröblacher, S., Jennewein, T., Vaziri, A., Weihs, G., Zeilinger, A.: Experimental quantum cryptography with qutrits. New J. Phys. 8, 75 (2006)

[30] Mafu, M., Dudley, A., Goyal, S., Giovannini, D., McLaren, M., Padgett, M.J., Konrad, T., Petruccione, F., Lütkenhaus, N., Forbes, A.: Higher-dimensional orbital-angular-momentum-based quantum key distribution with mutually unbiased bases. Phys. Rev. A 88, 032305 (2013)

[31] Ivanovic, I.D.: An inequality for the sum of entropies of unbiased quantum measurements. J. Phys. A: Math. Gen. 25, L363-L364 (1995)

[32] Sánchez, J.: Entropic uncertainty and certainty relations for complementary observables. Phys. Lett. A 173, 233-239 (1993)

[33] Rastegin, A.E.: Uncertainty and certainty relations for complementary qubit observables in terms of Tsallis' entropies. Quantum Inf. Process. 12, 2947-2963 (2013)

[34] Rastegin, A.E.: Uncertainty and certainty relations for Pauli observables in terms of Renyi entropies of order $\alpha \in(0 ; 1]$. Commun. Theor. Phys. 61, 293-298 (2014)

[35] Ballester, M.A., Wehner, S.: Entropic uncertainty and locking: tight bounds for mutually unbiased bases. Phys. Rev. A 75, 022319 (2007)

[36] Mandayam, P., Wehner, S., Balachandran, N.: A transform of complementary aspects with applications to entropic uncertainty relations. J. Math. Phys. 51, 082201 (2010)

[37] Bandyopadhyay, S., Mandayam, P.: Operational measure of incompatibility of noncommuting observables. Phys. Rev. A 87, $042120(2013)$

[38] Korzekwa, K., Lostaglio, M., Jennings, D., Rudolph, T.: Quantum and classical entropic uncertainty relations. Phys. Rev. A 89, $042122(2014)$

[39] Rastegin, A.E.: Uncertainty relations for MUBs and SIC-POVMs in terms of generalized entropies. Eur. Phys. J. D 67, $269(2013)$

[40] Helstrom, C.W.: Quantum Detection and Estimation Theory. (New York: Academic, 1976)

[41] Davies, E.B.: Information and quantum measurement. IEEE Trans. Inf. Theory 24, 596-199 (1978)

[42] Ban, M.: Discrimination among quantum states. Int. J. Theor. Phys. 43, 27-33 (2004)

[43] Ekert, A.K., Huttner, B., Palma, G.M., Peres A.: Eavesdropping on quantum cryptographical systems. Phys. Rev. A 50, 
1047-1056 (1994)

[44] Ivanovic, I.D.: How to differentiate between non-orthogonal states. Phys. Lett. A 123, 257-259 (1987)

[45] Dieks, D.: Overlap and distinguishability of quantum states. Phys. Lett. A 126, 303-306 (1988)

[46] Peres, A.: How to differentiate between non-orthogonal states. Phys. Lett. A 128, 19 (1988)

[47] Rastegin, A.E.: No-cloning theorem for a single POVM. Quantum Inf. Comput. 10, 0971-0980 (2010)

[48] Bennett, C.H.: Quantum cryptography using any two nonorthogonal states. Phys. Rev. Lett. 68, 3121-3124 (1992)

[49] Brierley, S., Weigert, S., Bengtsson, I.: All mutually unbiased bases in dimensions two to five. Quantum Inf. Comput. 10, 0803-0820 (2010)

[50] Kurzyński, P., Kaszub, W., Czechlewski, M.: Mutually unbiased bases and complementary spin 1 observables. J. Phys. A: Math. Theor. 43, 265303 (2010) 